\title{
Differential Induction of Stearoyl-CoA Desaturase 1 and 2 Genes by Fibrates in the Liver of Rats
}

\author{
Tohru Yamazaki, ${ }^{a}$ Hitomi Okada, ${ }^{a}$ Takeshi Sakamoto, ${ }^{a}$ Katsuyoshi Sunaga,${ }^{a}$ Tadashi Tsuda,${ }^{a}$ \\ Atsushi Mitsumoto, ${ }^{b}$ Naomi Kudo, ${ }^{a}$ and Yoichi Kawashima*,a \\ ${ }^{a}$ Faculty of Pharmaceutical Sciences, Josai University; 1-1 Keyakidai, Sakado, Saitama 350-0295, Japan: and \\ ${ }^{b}$ Faculty of Pharmaceutical Sciences, Josai International University; 1 Gumyo, Togane, Chiba 283-8555, Japan. \\ Received July 16, 2011; accepted October 28, 2011; published online November 2, 2011
}

\begin{abstract}
The administration of fibrates (fenofibrate, bezafibrate and clofibric acid) to rats induced stearoyl-CoA desaturase (SCD) in the liver, and increased relative expression of mRNAs encoding SCD1 and SCD2 in dose- and time-dependent manners. The magnitudes of the increases in SCD2 mRNA level caused by fenofibrate and clofibric acid were much higher than those of SCD1 at relatively higher doses of the fibrates, and a relatively long time ( 7 or $14 \mathrm{~d}$ ) was required for significant induction of SCD2 $\mathrm{mRNA}$ expression compared with that of SCD1. Although the absolute number of transcripts for SCD2 was 1,800 times lower than that of SCD1 in the control liver, it was strikingly increased by fibrates. These results suggest that differential regulations operate for the gene expression between SCD1 and SCD2, and that the physiological significance of SCD2 is distinct from that of SCD1 in the liver.
\end{abstract}

Key words stearoyl-CoA desaturase; clofibric acid; fenofibrate; rat liver

Stearoyl-CoA desaturase (SCD) is located in the endoplasmic reticulum membrane of the liver, and works together with reduced nicotinamide adenine dinucleotide (NADH)-cytochrome $b 5$ reductase and cytochrome $b 5$ as the rate-limiting enzyme necessary for desaturation of saturated fatty acids to monounsaturated fatty acids. ${ }^{1)}$ Four isoforms of SCD (SCD1 to SCD4) have been identified in mice, ${ }^{2-4)}$ whereas two isoforms (SCD1 and SCD2) have been characterized in rats. ${ }^{5}$ Accelerated formation of oleic acid by inducing SCD in the liver of rats is known as one of the biochemical effects of clofibric acid. $\left.{ }^{6}\right)$ Although clofibrate has been shown to induce SCD1 gene expression in the liver of mice, ${ }^{7,8)}$ detailed information is not available about the effects of fibrates on SCD2 gene expression in the liver. The aim of this study is to clarify the effects of fibrates on the induction of gene expression of SCD isoforms in the liver of rats.

\section{MATERIALS AND METHODS}

Animals All animal studies complied with the recommendations of the Institutional Board for Animal Studies, Josai University. Male Wistar rats aged 8 weeks were fed on a standard diet (CE-2, Clea Japan) or a diet admixed with fenofibrate (Sigma, St. Louis, MO, U.S.A.), bezafibrate (Sigma) or clofibric acid (Sigma). For examining the dose-response effects, rats were fed on a diet containing various concentrations of fenofibrate $(0,0.025,0.05,0.1$ or $0.2 \%(\mathrm{w} / \mathrm{w}))$, bezafibrate $(0,0.025,0.05,0.1$ or $0.2 \%(\mathrm{w} / \mathrm{w}))$ or clofibric acid $(0,0.05,0.1,0.2$ or $0.3 \%(\mathrm{w} / \mathrm{w}))$ for $14 \mathrm{~d}$. In the time-course experiments, rats were fed on a diet containing $0.1 \%(\mathrm{w} / \mathrm{w})$ fenofibrate or $0.3 \%(\mathrm{w} / \mathrm{w})$ clofibric acid for $0,1,2,3,7$ or $14 \mathrm{~d}$. Under diethyl ether anesthesia, livers were excised. One part of the liver was frozen in liquid nitrogen and stored at $-80^{\circ} \mathrm{C}$ for the determination of mRNA. The other part of the liver was perfused with ice-cold saline and homogenized with 4 volumes of $0.25 \mathrm{M}$ sucrose $/ 1 \mathrm{~mm}$ ethylenediaminetetraacetic acid (EDTA)/10 mm Tris- $\mathrm{HCl}$ (pH7.4), and was used for preparing microsomes.

Measurement of Stearoyl-CoA Desaturase Activity $\mathrm{Mi}-$ crosomes from the liver were prepared by differential centrifugations as described previously. ${ }^{9}$ ) SCD activity in microsomes was measured spectrophotometrically as described previously, ${ }^{9)}$ as the rate constant for the stearoyl-CoA-stimulated re-oxidation of NADH-reduced cytochrome $b 5$. The rate constant was measured in the presence $(k)$ and in the absence $\left(k^{-}\right)$of stearoyl-CoA; the rate constant for SCD was given by $k^{+}=k-k^{-}$.

Reverse Transcription and Quantitative Real-Time Polymerase Chain Reaction (Real-Time PCR) Total RNA was prepared from the liver using QIAzol reagent and RNeasy kit (QIAGEN, Hilden, Germany). cDNA was synthesized from $500 \mathrm{ng}$ of total RNA with avian myeloblastosis virus reverse transcriptase (Takara, Shiga, Japan). Real-time PCR experiments were carried out using SYBR premix ExTaq (Takara). The amplification and detection were performed with an $\mathrm{ABI}$ PRISM 7500 sequence detection system (Applied Biosystems, Foster City, CA, U.S.A.). The following primers were used: SCD1 forward, 5'-TCA CCT TGA GAG AAG AAT TAG CA3'; SCD1 reverse, 5'-TTC CCA TTC CCT TCA CTC TGA-3'; SCD2 forward, 5'-TGC ACC CCC AGA CAC TTG TAA-3'; SCD2 reverse, 5'-GGA TGC ATG GAA ACG CCA TA-3'; $\beta$-actin forward, 5'-TGC AGA AGG AGA TTA CTG CC-3'; $\beta$-actin reverse, 5'-CGC AGC TCA GTA ACA GTC C-3'. The thermal cycling program was as follows: $10 \mathrm{~s}$ denaturation step at $95^{\circ} \mathrm{C}$ followed by 50 cycles of $5 \mathrm{~s}$ denaturation at $95^{\circ} \mathrm{C}, 34 \mathrm{~s}$ annealing and extension at $60^{\circ} \mathrm{C}$. After the reaction, dissociation curve analyses were carried out to confirm the amplification of a single PCR product. Changes in gene expression were calculated by using the comparative threshold cycle $\left(C_{t}\right)$ method. $C_{\mathrm{t}}$ values were first normalized by subtracting the $C_{\mathrm{t}}$ value obtained from $\beta$-actin (control). Conventional PCR was performed with ExTaq polymerase (Takara).

For quantitation of each transcript, PCR products were purified and subcloned into the PCR2.1 plasmid using a TOPO TA cloning kit (Invitrogen, Carlsbad, CA, U.S.A.), and each transcript was determined using a standard curve of plasmid DNA.

Statistical Analysis Data were analyzed by one-way 
analysis of variance (ANOVA) followed by Scheffés multiple range test as a post hoc test. The results were considered to be significant if the value of $p$ was $<0.05$. Statistical significance between two groups was determined by Student's $t$-test.

\section{RESULTS}

Dose- and Time-Dependent Elevation of SCD1 and SCD2 mRNA by Fibrates SCD activities in hepatic microsomes of rats that were fed on diets containing various concentrations of one of the fibrates for $14 \mathrm{~d}$ were estimated (Figs. 1A, C, E). The three fibrates used in this study increased SCD activity in dose-dependent manners. At relatively low doses, fenofibrate up-regulated SCD activity more strongly than did clofibric acid. Treatments of rats with fenofibrate significantly increased the relative expression of mRNAs encoding SCD1 and SCD2 in dose-dependent manners (Fig. 1B). The relative expression of SCD1 mRNA increased with increasing dose and almost reached a plateau at a dose of fenofibrate of more than $0.05 \%$, whereas the relative expression of mRNA encoding SCD2 increased with the elevation of the dose of fenofibrate up to $0.2 \%$. Moreover, the extents of the increase in relative expression of SCD2 mRNA were strikingly higher than those of SCD1 at relatively high doses of fenofibrate. Namely, at the dietary concentration of $0.2 \%$ fenofibrate, relative expressions of SCD1 and SCD2 were 48 and 170 times those of the control, respectively (Fig. 1B). Similarly, but to a somewhat lesser extent, bezafibrate also significantly increased the relative expression of mRNAs encoding both the SCD isoforms (Fig. 1D). It was noteworthy, however, that there was some difference in the mode of action for the inductions of SCD1 and SCD2 mRNAs between clofibric acid and fenofibrate. Namely, clofibric acid induced relative expression of SCD1 mRNA to the same extent as fenofibrate, whereas clofibric acid induced gene expression of SCD2 less effectively than did fenofibrate (Figs. 1B, F).
A

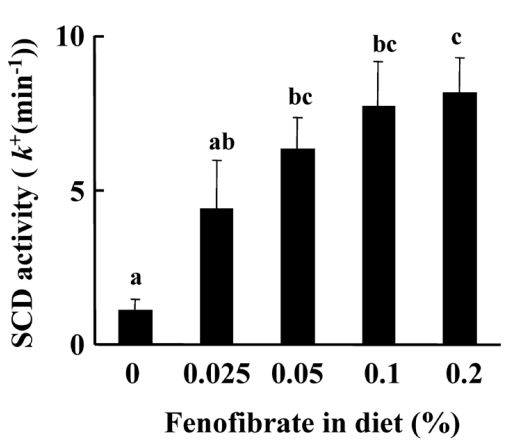

C

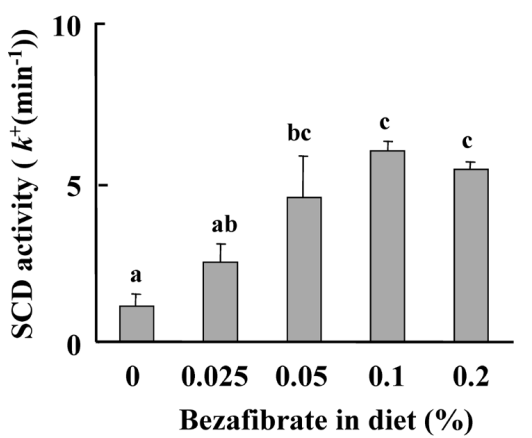

E

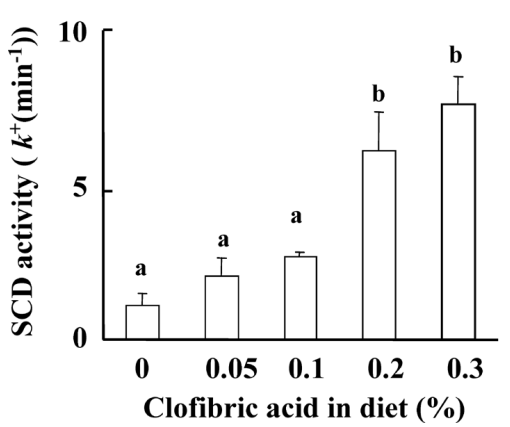

B

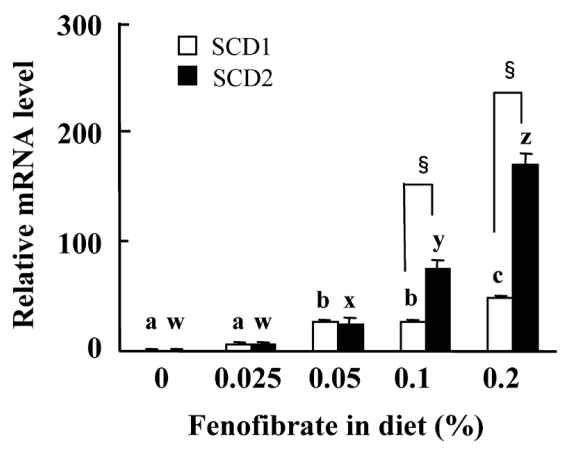

D
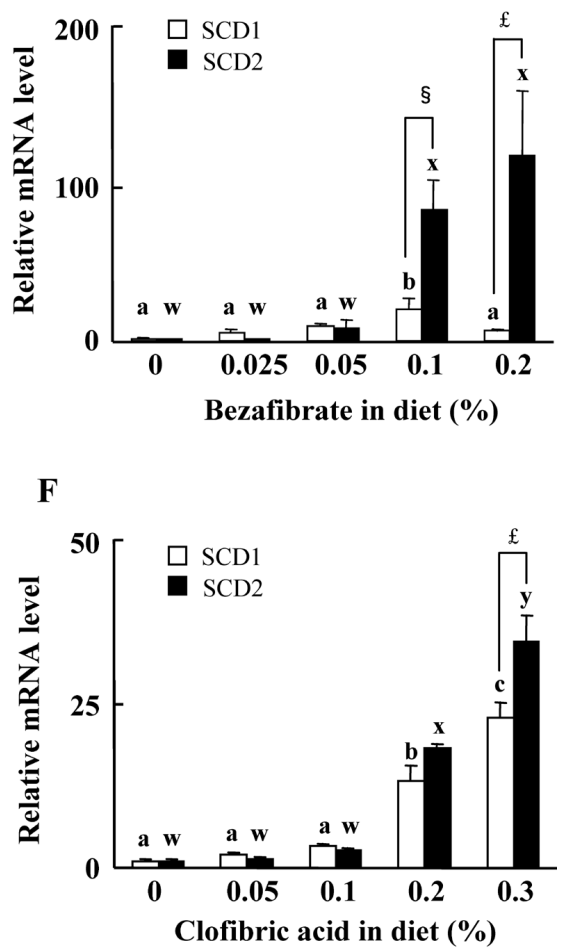

Fig. 1. Effects of Fibrates on SCD Activity and Relative Expression of mRNAs for SCD1 and SCD2 in the Liver of Rats

Rats were fed on the standard diet or a diet admixed with fenofibrate (A,B), bezafibrate (C,D) or clofibric acid (E,F) for $14 \mathrm{~d}$. Values are mean \pm S.D. for four rats. ${ }^{\mathrm{a}-\mathrm{c}}$ Differences in the mean without a common superscript are statistically significant $(p<0.05)$. ${ }^{\mathrm{w}-\mathrm{z}}$ Differences in the mean without a common superscript are statistically significant $(p<0.05)$. Significant difference in the two means between the corresponding dose points is represented as ${ }^{\mathrm{f}} p<0.05 ;{ }^{8} p<0.01$. 
The treatments with fenofibrate and clofibric acid increased the relative expression of mRNAs encoding SCD1 and SCD2 in time-dependent manners (Fig. 2). The relative expression of SCD1 mRNA in the liver of rats, which were fed on a diet containing $0.1 \%$ fenofibrate, gradually increased. On the other hand, there was a time lag until the induction of SCD2 mRNA expression and a clear increase in the relative expression of SCD2 was observed on day 7 (Fig. 2A). Similar but less pronounced inductions of mRNAs encoding SCD1 and SCD2 were brought about by clofibric acid (Fig. 2B). Thus, the induction of SCD2 mRNA seems to require more time and a higher dose of fibrates than that of SCD1 mRNA.

Effects of Fibrates on the Number of Transcript of SCD1 and SCD2 in the Liver The present study showed that the numbers of transcripts of SCD1 and SCD2 were $844.5 \pm 248.8$ and $0.45 \pm 0.10$, respectively, relative to $\beta$-actin; the copy number of SCD2 was about 1800 times lower than that of SCD1 in the liver of control rats (Fig. 3). The gene expression of SCD2 was drastically induced by the treatments of rats with more than $0.1 \%$ fenofibrate or $0.3 \%$ clofibric acid for $14 \mathrm{~d}$ (Fig. 3), although the numbers of transcripts of SCD2 at dietary concentrations of fenofibrate of 0.1 and $0.2 \%$ were still 180 and 59 times lower than those of SCD1, respectively (Figs. 3A, B).

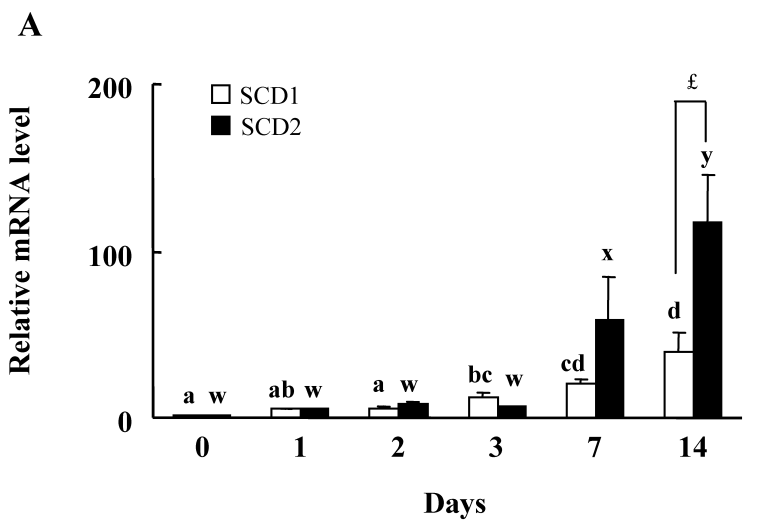

B

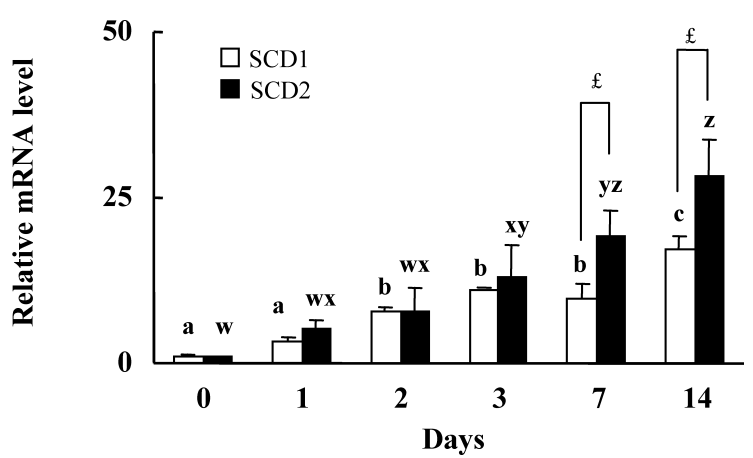

Fig. 2. Time Course for Induction of mRNAs Encoding SCD1 and SCD2 by Fibrates in the Liver of Rats

Rats were fed on a diet containing $0.1 \%(\mathrm{w} / \mathrm{w})$ fenofibrate (A) or $0.3 \%(\mathrm{w} / \mathrm{w})$ clofibric acid (B) for the indicated time. Values are mean \pm S.D. for four rats.

${ }^{\mathrm{a}-\mathrm{d}}$ Differences in the mean without a common superscript are statistically significant $(p<0.05) .{ }^{\mathrm{w}-\mathrm{z}}$ Differences in the mean without a common superscript are statistically significant $(p<0.05)$. Significant difference in the two mean between the corresponding time points was represented as ${ }^{f} p<0.05$.

\section{DISCUSSION}

Previous studies independently demonstrated that the administration of clofibarte to mice induced the level of SCD1 mRNA in the liver. ${ }^{7,8)}$ By contrast, in the course of our present study, results of analyzing DNA microarray were opened as the database, ${ }^{10)}$ which showed that SCD1 mRNA level in the liver was almost unchanged by repeated administration of clofibrate or fenofibrate at a dose of 300 or $1000 \mathrm{mg} / \mathrm{kg}$ of body weight, respectively, to rats for 4 to $29 \mathrm{~d}$. The present study have showed, however, that the three fibrates considerably induced SCD1 mRNA in the liver of rats. These results are in accordance with the previous findings of Diczfalusy et $a l^{7)}$ and Miller and Ntambi. ${ }^{8)}$ With regard to the physiological significance of SCD1 expression in the liver, a previous study provided an attractive hypothesis that SCD1 is colocalized with diacylglycerol acyltransferase 2 in the endoplasmic reticulum and that oleic acid, which is synthesized by SCD1, is channeled to diacylglycerol acyltransferase 2 for triacylglycerol synthesis. ${ }^{11)}$ Namely, SCD1 is considered to play a pivotal role in the regulation of hepatic and plasma triacylglycerol accumulation. It seems likely, therefore, that the SCD1, which was induced by fibrates, increasingly produced oleic acid that was incorporated into triacylglycerol by the action of diacylglycerol acyltransferase 2. In fact, proportion of oleic acid in triacylglycerol in the liver has been shown to increase considerably by the administration of clofibric acid to rats. ${ }^{12)}$

The present study showed that the transcript level of SCD2 was 1800 times lower than that of SCD1 in the liver of control rats. To our knowledge, this is the first evidence showing the exact number of transcript of SCD2 that were expressed in the liver of rats. The previous studies provided evidence showing that the mRNA level of SCD2 in the liver of mice or rat is extremely low or not detectable, ${ }^{7,13)}$ and, recently, transcript level of SCD2 has been shown to be 84 to 462 times lower than that of SCD1 in the liver of mice. ${ }^{14)}$ Accordingly, transcript level of SCD2 relative to that of SCD1 in the liver of rats are significantly lower than that of mice. It is noted that SCD2 is predominantly expressed in the brain, ${ }^{7)}$ the peripheral nervous system, ${ }^{15)}$ the testis, ${ }^{16)}$ the ovary, ${ }^{13)}$ brown adipose tissue, ${ }^{7)}$ the skin (embryo and neonates) ${ }^{17)}$ and the liver (embryo and neonates). ${ }^{17)}$ However, physiological significance and regulation of SCD2 in the liver has not been clarified yet. Moreover, the effects of fibrates on the expression of SCD2 gene have been little studied, despite the fact that a few studies have shown that the relative expressions of SCD1 mRNA in the liver were enhanced by the administration of clofibrate to mice., ${ }^{78}$ The database, which was opened in the course of this study, showed that the expressions of SCD in the liver were induced by the repeated administration of fenofibrate or clofibrate to rats, ${ }^{10)}$ but the extent of the induction was slight and the probe sets for SCD were thought not to be able to distinguish SCD1 and SCD2. Therefore, although the results of the database suggest the induction of SCD2 expression by the fibrates, the information was incomplete. Our present study showed that the transcript level of SCD2 in the liver of rats was strikingly induced by the administration of the three fibrates. It is considered that fibrates induce the mRNA expression of SCD1 in the liver of rats, because the peroxisome proliferator responsive element (PPRE)-like sequence exists in its promoter region. ${ }^{8)}$ On the other hand, the PPRE-like sequence was not confirmed 
A

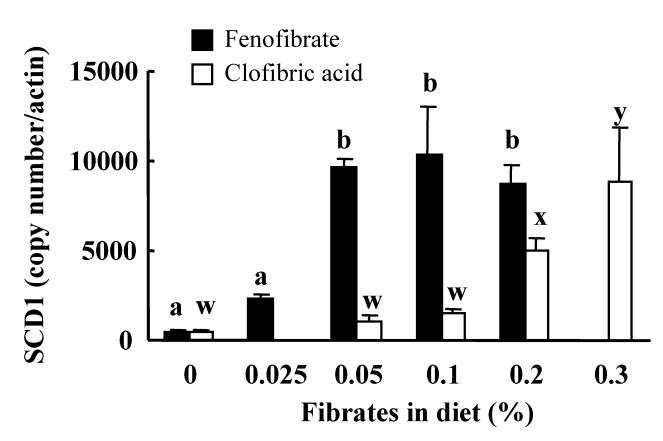

C

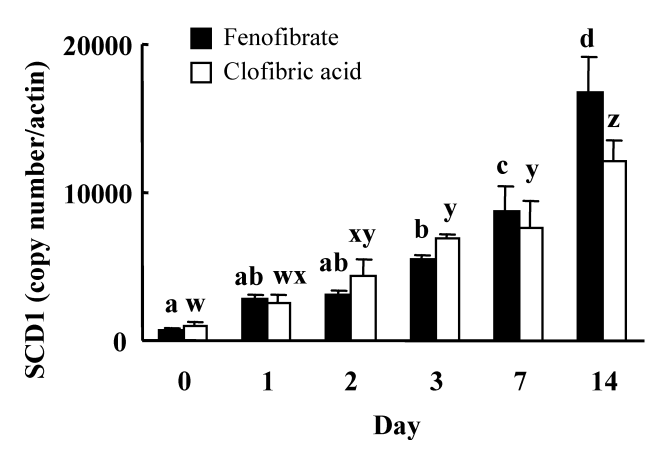

B

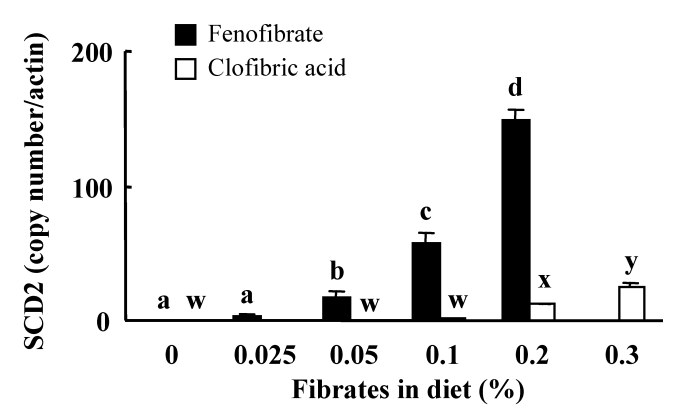

D

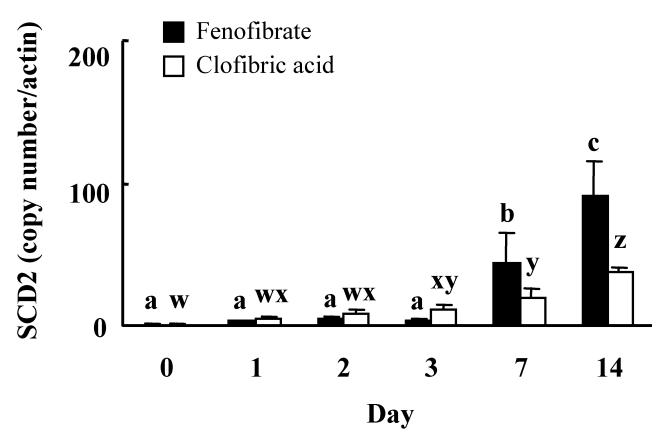

Fig. 3. Transcript Levels of SCD1 and SCD2 in the Liver of Rats That Were Treated with Fenofibrate or Clofibric Acid

Values are mean \pm S.D. for four rats and relative to $\beta$-actin. Using the calibration curve, cDNA concentration and copy number were calculated from the threshold cycle values for each SCD isoforms in the liver. (A) Dose-response for SCD1 mRNA; (B) dose-response for SCD2 mRNA; (C) time-course for SCD1 mRNA; (D) time-course for SCD2 mRNA. ${ }^{a-d}$ Differences in the mean without a common superscript are statistically significant $(p<0.05)$. ${ }^{\text {w-z }}$ Differences in the mean without a common superscript are statistically significant $(p<0.05)$.

in the promoter region ( -900 bases) of rat SCD2. Moreover, the present study demonstrated that induction of SCD2 mRNA requires more time and a higher dose of fibrates than that of SCD1 mRNA. Taken together, it seems likely that the induction of SCD2 is the secondary effect of fibrates through the activation of peroxisome proliferator-activated receptor alpha $(\operatorname{PPAR} \alpha)$ or the effects of fibrates beside PPAR $\alpha$. Thus, the present results suggest that the expression of the SCD2 gene is under the regulation that differs from that which operates for SCD1 gene expression, and that the physiological significance of the induced SCD2 is distinct from that of SCD1. Our previous study showed that clofibric acid induced lysophosphatidylcholine acyltransferase 3 in the liver of rats, ${ }^{18)}$ resulting in a striking increase in mass of palmitoyl-oleoyl-phosphatidylcholine. ${ }^{19)}$ It is of interest, therefore, to suggest the possibility that the SCD2 induced by fibrates is involved in the formation of palmitoyl-oleoyl-phosphatidylcholine, but not triacylglycerol.

In summary, the present study showed that the gene expressions of SCD1 and SCD2 are differentially regulated by fibrates, that SCD2 gene is expressed at extremely low levels in control liver, but is induced by fibrates more strikingly than SCD1 gene, and that fenofibrate up-regulates SCD2 more effectively than does clofibric acid, whereas fenofibrate and clofibric acid induce the expression of SCD1 mRNA to almost the same extent. These results, taken together, suggest the possibility that the physiological role of SCD2 is distinct from that of SCD1 in the liver.
Acknowledgement This work was partly supported by a Grant-in-Aid for Scientific Research from the Ministry of Education, Culture, Sport, Science and Technology of Japan.

\section{REFERENCES}

1) Strittmatter P, Spatz L, Corcoran D, Rogers MJ, Setlow B, Redline R. Purification and properties of rat liver microsomal stearyl coenzyme A desaturase. Proc. Natl. Acad. Sci. U.S.A., 71, 4565-4569 (1974).

2) Kaestner KH, Ntambi JM, Kelly TJ Jr, Lane MD. Differentiationinduced gene expression in 3T3-L1 preadipocytes. A second differentially expressed gene encoding stearoyl-CoA desaturase. J. Biol. Chem., 264, 14755-14761 (1989).

3) Zheng Y, Prouty SM, Harmon A, Sundberg JP, Stenn KS, Parimoo S. Scd3 - a novel gene of the stearoyl-CoA desaturase family with restricted expression in skin. Genomics, 71, 182-191 (2001).

4) Miyazaki M, Jacobson MJ, Man WC, Cohen P, Asilmaz E, Friedman JM, Ntambi JM. Identification and characterization of murine $\mathrm{SCD} 4$, a novel heart-specific stearoyl-CoA desaturase isoform regulated by leptin and dietary factors. J. Biol. Chem., 278, 3390433911 (2003).

5) Mihara K. Structure and regulation of rat liver microsomal stearoylCoA desaturase gene. J. Biochem., 108, 1022-1029 (1990).

6) Kawashima Y, Kozuka H. Increased activity of stearoyl-CoA desaturation in liver from rat fed clofibric acid. Biochim. Biophys. Acta, 713, 622-628 (1982).

7) Diczfalusy U, Eggertsen G, Alexson SEH. Clofibrate treatment increases stearoyl-CoA desaturase mRNA level and enzyme activity in mouse liver. Biochim. Biophys. Acta, 1259, 313-316 (1995). 
8) Miller CW, Ntambi JM. Peroxisome proliferators induce mouse liver stearoyl-CoA desaturase 1 gene expression. Proc. Natl. Acad. Sci. U.S.A., 93, 9443-9448 (1996).

9) Imai K, Koyama M, Kudo N, Shirahata A, Kawashima Y. Increase in hepatic content of oleic acid induced by dehydroepiandrosterone in the rat. Biochem. Pharmacol., 58, 925-933 (1999).

10) National Institute of Biomedical Innovation. "Open TG-GATES.": 〈http://toxico.nibio.go.jp〉, cited 15 June, 2011

11) Man WC, Miyazaki M, Chu K, Ntambi JM. Colocalization of SCD1 and DGAT2: implying preference for endogenous monounsaturated fatty acids in triglyceride synthesis. J. Lipid Res., 47, 1928-1939 (2006).

12) Kawashima Y, Hirose A, Kozuka H. Modification by clofibric acid of acyl composition of glycerolipids in rat liver. Possible involvement of fatty acid chain elongation and desaturation. Biochim. Biophys. Acta, 795, 543-551 (1984).

13) Moreau C, Froment P, Tosca L, Moreau V, Dupont J. Expression and regulation of the SCD2 desaturase in the rat ovary. Biol. Reprod., 74, 75-87 (2006).

14) $\mathrm{Xu} \mathrm{M}$, Wang $\mathrm{W}$, Frontera JR, Neely MC, Lu J, Aires D, Hsu FF, Turk J, Swerdlow RH, Carlson SE, Zhu H. Ncb5or deficiency in- creases fatty acid catabolism and oxidative stress. J. Biol. Chem., 286, 11141-11154 (2011).

15) Garbay B, Boiron-Sargueil F, Shy M, Chbihi T, Jiang H, Kamholz J, Cassagne C. Regulation of oleoyl-CoA synthesis in the peripheral nervous system: demonstration of a link with myelin synthesis. $J$. Neurochem., 71, 1719-1726 (1998).

16) Saether T, Tran TN, Rootwelt H, Christophersen BO, Haugen TB. Expression and regulation of delta5-desaturase, delta6-desaturase, stearoyl-coenzyme A (CoA) desaturase 1, and stearoyl-CoA desaturase 2 in rat testis. Biol. Reprod., 69, 117-124 (2003).

17) Miyazaki M, Dobrzyn A, Elias PM, Ntambi JM. Stearoyl-CoA desaturase-2 gene expression is required for lipid synthesis during early skin and liver development. Proc. Natl. Acad. Sci. U.S.A., 102, $12501-12506$ (2005).

18) Yamazaki T, Hirose A, Sakamoto T, Okazaki M, Mitsumoto A, Kudo N, Kawashima Y. Peroxisome proliferators attenuate free arachidonic acid pool in the kidney through inducing lysophospholipid acyltransferases. J. Pharmacol. Sci., 111, 201-210 (2009).

19) Mizuguchi H, Kudo N, Kawashima Y. Metabolic alterations by clofibric acid in the formation of molecular species of phosphatidylcholine in rat liver. Biochem. Pharmacol., 62, 853-861 (2001). 